\title{
SOME FAMILIES OF MEROMORPHIC $p$ - VALENT FUNCTIONS INVOLVINGA NEW OPERATOR DEFINED BY GENERALIZED MITTAG-LEFFLER FUNCTION
}

M. K. Aouf* and T. M. Seoudy

* Department of Mathematics, Faculty of Science, Mansoura University, Mansoura 35516, Egypt mkaouf127@yahoo.com

Department of Mathematics, Faculty of Science, Fayoum University, Fayoum 63514, Egypt tms00@fayoum.edu.eg

Receive 10/2/2018, Revised, Accepted 21/5/2018

Abstract. In this paper, we introduce some classes of meromorphic multivalent functions which are defined by a new linear operator containing the generalized Mittag-Leffler function. Using convolution results obtained by Sarkar et al . [1] (see also Mostafa and Aouf [2]), we investigate some properties and inclusion relations for functions in these classes.

2010 Mathematics Subject Classification. 30C45, 30C80, 33E12.

Key Words and Phrases. subordination, meromorphic $p$ - valent functions, Mittag-Leffler function, convolution, linear operator.

\section{Introduction}

Let $\sum_{p}$ be the class of functions of the form:

$$
f(z)=z^{-p}+\sum_{n=1}^{\infty} a_{n} z^{n-p} \quad(p \in \mathbb{N}=\{1,2, \ldots\}),
$$

which are analytic and $p$-valent in the punctured unit disk $\mathbb{U}^{*}=\mathbb{U} \backslash\{0\}$, where $\mathbb{U}=\{z: z \in \mathbb{C},|z|<1\}$. If $f$ and $g$ are analytic functions in $\mathbb{U}$, we say that $f$ is subordinate to $g$, written $f \prec g$ if there exists a Schwarz function $w$, which (by definition) is analytic in $\mathbb{U}$ with $w(0)=0$ and $|w(z)|<1$ for all $z \in \mathbb{U}$, such that $f(z)=g(w(z)), z \in \mathbb{U}$. Furthermore, if the function $g$ is univalent in $\mathbb{U}$, then we have the following equivalence (see [3] and [4]):

$$
f(z) \prec g(z) \Leftrightarrow f(0)=g(0) \text { and } f(U) \subset g(U) .
$$

For functions $f$ given by (1.1) and $g \in \sum_{p}$ given by

$$
g(z)=z^{-p}+\sum_{n=1}^{\infty} b_{n} z^{n-p},
$$

the Hadamard product (or convolution) of $f$ and $g$ is defined by

$$
(f * g)(z)=z^{-p}+\sum_{n=1}^{\infty} a_{n} b_{n} z^{n-p}=(g * f)(z) .
$$

The Mittag-Leffler function $E_{\alpha}(z)(z \in \mathbb{C})$ is defined by (see [5] and [6]):

$$
E_{\alpha}(z)=\sum_{n=0}^{\infty} \frac{z^{n}}{\Gamma(\alpha n+1)}(\alpha \in \mathbb{C}, \Re\{\alpha\}>0) .
$$

Srivastava and Tomovski [7] introduced the generalized Mittag-Leffler function $E_{\alpha, \beta}^{\gamma, k}(z)(z \in \mathbb{C})$ in the form:

$$
E_{\alpha, \beta}^{\gamma, k}(z)=\sum_{n=0}^{\infty} \frac{(\gamma)_{n k} z^{n}}{\Gamma(\alpha n+\beta) n !}
$$


where $\beta, \gamma \in \mathbb{C}, \Re\{\alpha\}>\max \{0 ; \Re\{k\}-1\} ; \Re\{k\}>0, \Re\{\alpha\}=0$ when $\Re\{k\}=1$ with $\beta \neq 0$ and $(v)_{m}$ denotes the Pochhammer symbol (or the shifted factorial) defined by

$$
(\nu)_{m}:=\left\{\begin{array}{lll}
1, & \text { if } & m=0 \\
\nu(\nu+1)(\nu+2) \ldots(\nu+m-1), & \text { if } & m \in \mathbb{N}
\end{array}\right.
$$

Srivastava and Tomovski [7] proved that the function $E_{\alpha, \beta}^{\gamma, k}(z)$ defined by (1.4) is an entire function in the complex $z$-plane We now define the function $\mathcal{B}_{p, \alpha, \beta}^{\gamma, k}(z)$ by

$$
\mathcal{B}_{p, \alpha, \beta}^{\gamma, k}(z)=z^{-p} \Gamma(\beta) E_{\alpha, \beta}^{\gamma, k}(z)
$$

Corresponding to the function $\mathcal{B}_{p, \alpha, \beta}^{\gamma, k}(z)$ defined by (1.5), we introduce a linear operator $\mathcal{T}_{p, \alpha, \beta}^{\gamma, k} f(z): \sum_{p} \rightarrow \sum_{p}$ by

$$
\begin{gathered}
\mathcal{T}_{p, \alpha, \beta}^{\gamma, k} f(z)=\mathcal{B}_{p, \alpha, \beta}^{\gamma, k}(z) * f(z)=z^{-p}+\sum_{n=1}^{\infty} \frac{\Gamma(\gamma+n k) \Gamma(\beta)}{\Gamma(\gamma) \Gamma(\beta+\alpha n) n !} a_{n} z^{n-p} \\
(\Re\{\alpha\}>\max \{0 ; \Re\{k\}-1\} ; \Re\{k\}>0, \Re\{\alpha\}=0 \text { when } \Re\{k\}=1 \text { with } \beta \neq 0) .
\end{gathered}
$$

We note that

$$
\mathcal{T}_{p, 0, \beta}^{1,1} f(z)=f(z) \quad \text { and } \quad \mathcal{T}_{p, 0, \beta}^{2,1} f(z)=z f^{\prime}(z)+(p+1) f(z)
$$

Also, it is easily verified from (1.6) that

$$
k z\left(\mathcal{T}_{p, \alpha, \beta}^{\gamma, k} f(z)\right)^{\prime}=\gamma \mathcal{T}_{p, \alpha, \beta}^{\gamma+1, k} f(z)-(\gamma+p k) \mathcal{T}_{p, \alpha, \beta}^{\gamma, k} f(z)
$$

and

$$
\alpha z\left(\mathcal{T}_{p, \alpha, \beta+1}^{\gamma, k} f(z)\right)^{\prime}=\beta \mathcal{T}_{p, \alpha, \beta}^{\gamma, k} f(z)-(\beta+p \alpha) \mathcal{T}_{p, \alpha, \beta+1}^{\gamma, k} f(z)
$$

For $-1 \leq A<B \leq 1$ and $z \in \mathbb{U}^{*}$, Mogra [8] defined the following subclass of $\sum_{p}$ as follows

$$
\sum S_{p}[A, B]=\left\{f \in \sum_{p}:-\frac{z f^{\prime}(z)}{f(z)} \prec p \frac{1+A z}{1+B z}, z \in \mathbb{U}\right\}
$$

and Srivastava et al. [9] defined the following subclass of $\sum_{p}$ as follows

$$
\sum K_{p}[A, B]=\left\{f \in \sum_{p}:-\left[1+\frac{z f^{\prime \prime}(z)}{f^{\prime}(z)}\right] \prec p \frac{1+A z}{1+B z}, z \in \mathbb{U}\right\}
$$

It is clear that

$$
f(z) \in \sum K_{p}[A, B] \Leftrightarrow-\frac{z f^{\prime}(z)}{p} \in \sum S_{p}[A, B]
$$

We note that

(i) $\sum S_{1}[2 \alpha-1,1]=\sum S^{*}(\alpha)(0 \leq \alpha<1)$ (Juneja and Reddy [10]),

(ii) $\sum K_{1}[2 \alpha-1,1]=\sum K(\alpha)(0 \leq \alpha<1)$ (Srivastava et al. [9]),

(iii) $\sum S_{p}\left[\frac{2 \alpha}{p}-1,1\right]=\sum S_{p}^{*}(\alpha)(0 \leq \alpha<p)$ (Aouf and Hossen [11]),

(iv) $\sum K_{p}\left[\frac{2 \alpha}{p}-1,1\right]=\sum K_{p}(\alpha)(0 \leq \alpha<p)$ (Aouf and Srivastava [12]).

Using the operator $\mathcal{T}_{p, \alpha, \beta}^{\gamma, k}$, we define the classes $\sum S_{p, \alpha, \beta}^{\gamma, k}(A, B)$ and $\sum K_{p, \alpha, \beta}^{\gamma, k}(A, B)$ as follows:

$$
\sum S_{p, \alpha, \beta}^{\gamma, k}(A, B)=\left\{f \in \sum_{p}: \mathcal{T}_{p, \alpha, \beta}^{\gamma, k} f(z) \in \sum S_{p}[A, B], z \in \mathbb{U}^{*}\right\},
$$


and

$$
\sum K_{p, \alpha, \beta}^{\gamma, k}(A, B)=\left\{f \in \sum_{p}: \mathcal{T}_{p, \alpha, \beta}^{\gamma, k} f(z) \in \sum K_{p}[A, B], z \in \mathbb{U}^{*}\right\}
$$

We notice that

$$
f \in \sum K_{p, \alpha, \beta}^{\gamma, k}(A, B) \Leftrightarrow-\frac{z f^{\prime}}{p} \in \sum S_{p, \alpha, \beta}^{\gamma, k}(A, B) .
$$

To prove our results we need the following lemmas.

Lemma 1.1. [1,2] The function $f(z)$ defined by (1.1) is in the class $\sum S_{p}[A, B]$ if and only if

$$
z^{p}\left[f(z) * \frac{1+(D-1) z}{z^{p}(1-z)^{2}}\right] \neq 0
$$

where

$$
D=\frac{e^{-i \theta}+B}{p(A-B)}
$$

Lemma 1.2. [1,2] The function $f(z)$ defined by (1.1) is in the class $\sum K_{p}[A, B]$ if and only if

$$
z^{p}\left\{f(z) *\left[\frac{p-\{2+p-(p-1)(D-1)\} z-(p+1)(D-1) z^{2}}{p z^{p}(1-z)^{3}}\right]\right\} \neq 0 .
$$

Lemma 1.3. [13] Let $h$ be convex (univalent) in $\mathbb{U}$, with $\operatorname{Re}[\delta h(z)+\eta]>0$ for all $z \in \mathbb{U}$. If $g$ is analytic in $\mathbb{U}$, with $g(0)=h(0)$, then

$$
g(z)+\frac{z g^{\prime}(z)}{\delta g(z)+\eta} \prec h(z) \Rightarrow g(z) \prec h(z) .
$$

In this paper, we obtain some interesting results for the families $\sum S_{p, \alpha, \beta}^{\gamma, k}(A, B)$ and $\sum K_{p, \alpha, \beta}^{\gamma, k}(A, B)$ of meromorphic $p$-valent functions defined by the operator $\mathcal{T}_{p, \alpha, \beta}^{\gamma, k}$.

\section{Main results}

Unless otherwise mentioned, we shall assume in this paper that $\Re\{\alpha\}>\max \{0 ; \Re\{k\}-1\}, \Re\{k\}>0, \Re\{\alpha\}=0$ when $\Re\{k\}=1$ with $\beta \neq 0,-1 \leq A<B \leq 1,0<\theta<2 \pi, p \in \mathbb{N}$ and $z \in \mathbb{U}^{*}$.

Theorem 2.1. The function $f(z)$ defined by (1.1) is in the class $\sum S_{p, \alpha, \beta}^{\gamma, k}(A, B)$ if and only if

$$
1+\sum_{n=1}^{\infty}\left[\frac{n e^{-i \theta}+p A+(n-p) B}{p(A-B)}\right] \frac{\Gamma(\gamma+n k) \Gamma(\beta)}{\Gamma(\gamma) \Gamma(\beta+\alpha n) n !} a_{n} z^{n} \neq 0 .
$$

Proof. From Lemma 1.1, we find that $f \in \sum S_{p, \alpha, \beta}^{\gamma, k}(A, B)$ if and only if

$$
z^{p}\left[\mathcal{T}_{p, \alpha, \beta}^{\gamma, k} f(z) * \frac{1+(D-1) z}{z^{p}(1-z)^{2}}\right] \neq 0 .
$$

Expanding $\frac{1+(D-1) z}{z^{p}(1-z)^{2}}$, we have (2.1) which completes the proof of Theorem 2.1.

Theorem 2.2. The function $f(z)$ defined by (1.1) is in the class $\sum K_{p, \alpha, \beta}^{\gamma, k}(A, B)$ if and only if

$$
1-\sum_{n=1}^{\infty}\left[\frac{n\left[n e^{-i \theta}+p A+(n-p) B\right]}{p^{2}(A-B)}\right] \frac{\Gamma(\gamma+n k) \Gamma(\beta)}{\Gamma(\gamma) \Gamma(\beta+\alpha n) n !} a_{n} z^{n} \neq 0 .
$$


Proof. From Lemma 1.2, we find that $f \in \sum K_{p, \alpha, \beta}^{\gamma, k}(A, B)$ if and only if

$$
z^{p}\left\{\mathcal{T}_{p, \alpha, \beta}^{\gamma, k} f(z) *\left[\frac{p-\{2+p-(p-1)(D-1)\} z-(p+1)(D-1) z^{2}}{p z^{p}(1-z)^{3}}\right]\right\} \neq 0 .
$$

Now it can be easily shown that

$$
\begin{aligned}
z^{-p}(1-z)^{-3}=z^{-p} & +\sum_{n=1}^{\infty} \frac{(n+1)(n+2)}{2} z^{n-p}, \\
z^{1-p}(1-z)^{-3} & =\sum_{n=1}^{\infty} \frac{n(n+1)}{2} z^{n-p} \\
z^{2-p}(1-z)^{-3} & =\sum_{n=1}^{\infty} \frac{n(n-1)}{2} z^{n-p} .
\end{aligned}
$$

Using (2.4)-(2.6) and (1.16) in (2.3), we have the desired result which completes the proof of Theorem 2.2.

Theorem 2.3. If the function $f(z)$ defined by (1.1) belongs to the class $\sum S_{p, \alpha, \beta}^{\gamma, k}(A, B)$, then

$$
\sum_{n=1}^{\infty}[n+p A+(n-p) B]\left|\frac{\Gamma(\gamma+n k) \Gamma(\beta)}{\Gamma(\gamma) \Gamma(\beta+\alpha n) n !} a_{n}\right| \leq p(A-B) .
$$

Proof. Since

$$
\left|\frac{n e^{-i \theta}+p A+(n-p) B}{p(A-B)}\right|=\frac{\left|n e^{-i \theta}+p A+(n-p) B\right|}{p(A-B)} \leq \frac{n+p A+(n-p) B}{p(A-B)}
$$

and

$$
\begin{aligned}
& \left|1+\sum_{n=1}^{\infty} \frac{\left[n e^{-i \theta}+p A+(n-p) B\right]}{p(A-B)} \frac{\Gamma(\gamma+n k) \Gamma(\beta)}{\Gamma(\gamma) \Gamma(\beta+\alpha n) n !}\right| a_{n} z^{n}|| \\
& >1-\sum_{n=1}^{\infty}\left|\frac{\left[n e^{-i \theta}+p A+(n-p) B\right]}{p(A-B)}\right|\left|\frac{\Gamma(\gamma+n k) \Gamma(\beta)}{\Gamma(\gamma) \Gamma(\beta+\alpha n) n !} a_{k}\right| .
\end{aligned}
$$

The result follows from Theorem 2.1.

Using the same technique, we can also prove the following theorem.

Theorem 2.4. If the function $f(z)$ defined by (1.1) belongs to the class $\sum K_{p, \alpha, \beta}^{\gamma, k}(A, B)$, then

$$
\sum_{n=1}^{\infty} n[n+p A+(n-p) B]\left|\frac{\Gamma(\gamma+n k) \Gamma(\beta)}{\Gamma(\gamma) \Gamma(\beta+\alpha n) n !} a_{k}\right| \leq p^{2}(A-B)
$$

Theorem 2.5. Let the function $f(z)$ be defined by (1.1). If

$$
\frac{1+A B+(A+B) \cos \theta}{1+B^{2}+2 B \cos \theta} \leq \frac{\gamma}{k}+p
$$

and $f \in \sum S_{p, \alpha, \beta}^{\gamma+1, k}(A, B)$ with $\gamma, k>0$ and $\mathcal{T}_{p, \alpha, \beta}^{\gamma, k} f(z) \neq 0$, then $f \in \sum S_{p, \alpha, \beta}^{\gamma, k}(A, B)$.

Proof. Let $f \in \sum S_{p, \alpha, \beta}^{\gamma+1, k}(A, B)$ and define the function

$$
g(z)=-\frac{z\left(\mathcal{T}_{p, \alpha, \beta}^{\gamma, k} f(z)\right)^{\prime}}{\mathcal{T}_{p, \alpha, \beta}^{\gamma, k} f(z)}
$$


we see that $g(z)$ is analytic in $\mathbb{U}$ with $g(0)=1$. Using the identity (1.7) in (2.10) we have

$$
\frac{\gamma}{k} \frac{\mathcal{T}_{p, \alpha, \beta}^{\gamma+1, k} f(z)}{\mathcal{T}_{p, \alpha, \beta}^{\gamma, k} f(z)}=-g(z)+\frac{\gamma}{k}+p .
$$

Differentiating (2.11) logarithmically and using (2.10), we have

$$
-\frac{z\left(\mathcal{T}_{p, \alpha, \beta}^{\gamma+1, k} f(z)\right)^{\prime}}{\mathcal{T}_{p, \alpha, \beta}^{\gamma+1, k} f(z)}=g(z)+\frac{z g^{\prime}(z)}{-g(z)+\frac{\gamma}{k}+p} \prec \frac{1+A z}{1+B z}=h(z) .
$$

Simple computations show that the inequality $\Re\left\{-h(z)+\frac{\gamma}{k}+p\right\}>0$ can be written in the form

$$
\Re \frac{1+A z}{1+B z}<\frac{\gamma}{k}+p
$$

which is equivalent to $(2.9)$. Since the function $h(z)$ is a convex function, then applying Lemma 1.3, we see that the subordination (2.12) implies $g(z) \prec h(z)$. This completes the proof of Theorem 2.5.

Theorem 2.5 yields the following theorem.

Theorem 2.6. Let the function $f(z)$ be defined by (1.1). If (2.9) holds and $f \in \sum K_{p, \alpha, \beta}^{\gamma+1, k}(A, B)$ with $\gamma, k>0$ and $\mathcal{T}_{p, \alpha, \beta}^{\gamma, k} f(z) \neq 0$, then $f \in \sum K_{p, \alpha, \beta}^{\gamma, k}(A, B)$.

Proof. Let $f \in \sum K_{p, \alpha, \beta}^{\gamma, k}(A, B)$ we have

$$
\begin{aligned}
f \in \sum K_{p, \alpha, \beta}^{\gamma, k}(A, B) & \Leftrightarrow \quad-\frac{z f^{\prime}}{p} \in \sum S_{p, \alpha, \beta}^{\gamma, k}(A, B) \quad \text { (From (1.14)) } \\
& \Rightarrow \quad-\frac{z f^{\prime}}{p} \in \sum S_{p, \alpha, \beta}^{\gamma+1, k}(A, B) \quad \text { (by Theorem 2.5) } \\
& \Leftrightarrow \quad f \in \sum K_{p, \alpha, \beta}^{\gamma+1, k}(A, B)
\end{aligned}
$$

this proves Theorem 2.6

Similarly we can prove the following theorems

Theorem 2.7. Let the function $f(z)$ be defined by (1.1). If

$$
\frac{1+A B+(A+B) \cos \theta}{1+B^{2}+2 B \cos \theta} \leq \frac{\beta}{\alpha}+p
$$

and $f \in \sum S_{p, \alpha, \beta}^{\gamma, k}(A, B)$ with $\alpha, \beta>0$ and $\mathcal{T}_{p, \alpha, \beta+1}^{\gamma, k} f(z) \neq 0$, then $f \in \sum S_{p, \alpha, \beta+1}^{\gamma, k}(A, B)$.

Theorem 2.8. Let the function $f(z)$ be defined by (1.1). If (2.13) holds and $f \in \sum K_{p, \alpha, \beta}^{\gamma, k}(A, B)$ with $\alpha, \beta>0$ and $\mathcal{T}_{p, \alpha, \beta+1}^{\gamma, k} f(z) \neq 0$, then $f \in \sum K_{p, \alpha, \beta+1}^{\gamma, k}(A, B)$.

\section{References}

[1] N. Sarkar, P. Goswami and and M. K. Aouf, Convolution properties for certain classes of meromorphic p-valent functions defined by subordination, Thai J. Math., 10 (2012), no. 3, 577-585.

[2] A. O. Mostafa and M. K. Aouf, On convolution properties for some classes of meromorphic functions associated with linear operator, Bull. Iranian Math. Soc., 41 (2015), no. 2, 325-332.

[3] S. S. Miller and P. T. Mocanu, Differential Subordinations: Theory and Applications, Series on Monographs and Textbooks in Pure and Appl. Math. No. 225, Marcel Dekker Inc., New York, 2000. 
[4] T. Bulboacă, Differential Subordinations and Superordinations. Recent Results, House of Scientific Book Publ., ClujNapoca, 2005.

[5] G. M. Mittag-Leffler, Sur la nouvelle function, C.R. Acad. Sci., Paris, 137(1903), 554-558.

[6] G. M. Mittag-Leffler, Sur la representation analytique d'une function monogene (cinquieme note), Acta Math., 29 (1905), 101-181.

[7] H. M. Srivastava and Z. Tomovski, Fractional calculus with an integral operator containing a generalized Mittag-Leffler function in the kernal, Appl. Math. Comp., 211(2009), 198-210.

[8] M. L. Mogra, Meromorphic multivalent functions with positive coefficients I, Math. Japon., 35 (1990), 1-11.

[9] H. M. Srivastava, H. M. Hossen and M. K. Aouf, A unified presentation of some classes of meromorphically multivalent functions, Comput. Math. Appl., 38 (1999), 63-70.

[10] O. P. Juneja and T. R. Reddy, Meromorphic starlike univalent functions with positive coefficients, Ann. Univ. Mariae Curie-Sklod., 39 (1985), no. 9, 65-75.

[11] M. K. Aouf and H. M. Hossen, New certeria for meromorphic $p$-valent starlike functions, Tsukuba J. Math., 17 (1993), no. $2,481-486$.

[12] M. K. Aouf and H. M. Srivastava, A new criteria for meromorphicallyp-valent convex functions of order alpha, Math. Sci. Res. Hot-line, 1 (1997), no. 8, 7-12.

[13] P. J. Eenigenburg, S. S. Miller, P. T. Mocanu and M. O. Reade, Second order differential inequalities in the complex plane, J. Math. Anal. Appl., 65 (1978), 289-305. 